\title{
Impacto do perfil clínico e sociodemográfico na adesão ao tratamento de pacientes de um Centro de Atenção Psicossocial a Usuários de Álcool e Drogas (CAPSad)
}

Impact of clinical and socio-demographic profiles in treatment adherence of patients attending a day hospital for alcohol and drug abuse

Clayton Peixoto', Carlos Henrique de Oliveira Prado', Cristiele Pedroso Rodrigues', Julio Nelson Devicari Cheda', Letícia Brito Tavares da Mota', André Barciela Veras²

\section{RESUMO}

Objetivo: O presente estudo compara os perfis sociodemográfico e clínico de pacientes que aderiram ou que não aderiram ao tratamento no Centro de Atenção Psicossocial a Usuários de Álcool e Drogas (CAPSad). Método: Foram analisados 316 prontuários de pacientes do CAPSad de Campo Grande (MS). Resultados: Com isso, pudemos observar que o grupo de pacientes que aderem ao tratamento são mais comumente homens $(p=0,072)$, mais velhos

\section{Palavras-chave}

CAPSad, álcool, drogas, adesão terapêutica.

\section{Keywords}

CAPSad, alcohol, drugs, treatment dropout. $(p=0,008)$, registrados por pai e por mãe $(p=0,068)$, usuários de álcool $(p=0,005)$ e com maior tempo de uso $(p=0,005)$. Conclusão: Há a necessidade de reformulação das abordagens atualmente aplicadas aos usuários do CAPSad com perfil de não adesão ao tratamento.

\section{ABSTRACT}

Objective: The present study compares the sociodemografic and clinical profile of patients that remain or not in treatment in a specialized center for alcohol and drugs (CAPSad). Method: Three hundred and sixteen medical records of a specialized center in Campo Grande (MS) were evaluated. Results: Thus we observed that the group of patients that do not remain in the treatment are commonly men ( $p=0,072)$, elderly $(p=0,008)$, with father and mother record ( $p=0,068)$, alcoholic $(p=0,005)$ and with more time of addiction $(p=0,005)$. Conclusion: There is a need to reformulate the approaches applied today in CAPSad, especially among patients with predictors of treatment dropout.
1 Universidade para o Desenvolvimento do Estado e da Região do Pantanal (Anhanguera-Uniderp), Campo Grande, MS, Brasil. 2 Universidade Federal do Rio de Janeiro (UFRJ), Instituto de Psiquiatria, Laboratório de Pânico e Respiração, Conselho Nacional de Desenvolvimento Científico e Tecnológico (CNPq)

Endereço para correspondência: Clayton Peixoto Rua Carmem Miranda, 151, JAR - 79083-390 - Campo Grande, MS, Brasil E-mail: clayton.ps@hotmail.com 


\section{INTRODUÇÃO}

O uso abusivo de álcool e outras drogas é um fenômeno presente e preocupante em todo o mundo. Atualmente, estima-se que cerca de $10 \%$ da população urbanizada de todo o mundo faz uso abusivo de drogas e que o álcool sozinho é responsável por 3,2\% das mortes em todo o mundo 1,2.

Um relatório do Escritório das Nações Unidas sobre Drogas e Crimes indica que, no Brasil, 68,7\% da população consumiu álcool em algum momento da vida, e 11,2\% apresentam dependência. O referido relatório também descreve a prevalência de uso de outras drogas pela população brasileira entre 12 e 65 anos, da seguinte forma: opiácios - 0,5\%; cocaína 0,7\%; maconha - 2,6\%; anfetamina - 0,7\%; ecstasy - 0,2\%3.

Diante desse quadro, o governo brasileiro tem adotado, por meio de política do Ministério da Saúde, estratégias que visam combater o avanço do uso abusivo de álcool e de outras drogas ${ }^{4-6}$.

Entre essas estratégias está a implantação do Centro de Atenção Psicossocial a Usuários de Álcool e Drogas (CAPSad) em cidades com mais de 100 mil habitantes, com a finalidade de disponibilizar tratamento a pacientes que fazem uso prejudicial de álcool e outras drogas, por meio de uma proposta baseada em serviços comunitários e apoiada por leitos psiquiátricos em hospital geral de acordo com as necessidades dos pacientes. O CAPSad oferece as seguintes modalidades de tratamento: intensivo, semi-intensivo e não intensivo. Entende-se por tratamento intensivo a modalidade de atendimento direcionada a pessoas com grave sofrimento psíquico, que precisam de atenção contínua - nessa modalidade o paciente é atendido diariamente. $O$ tratamento semi-intensivo é uma modalidade oferecida a pacientes que necessitam de atenção direta da equipe, mas apresentam menos sofrimento e melhor estruturação psíquica do que no caso anterior - nessa modalidade o usuário pode ser atendido até 12 dias no mês. Já o tratamento não intensivo é oferecido a pacientes que não necessitam de suporte contínuo, limitando-se o seu atendimento em até três dias no mês. As atividades consistem em atendimento individual, em grupo, oficinas terapêuticas, visitas domiciliares, repouso e desintoxicação ambulatorial?.

Entretanto, apesar do esforço do governo nesse sentido, ainda é grande o número de pacientes que desistem antes do término do tratamento ${ }^{8,9}$. Tal ocorrência é altamente prejudicial às políticas direcionadas aos usuários de álcool e outras drogas, uma vez que a eficácia de qualquer tratamento depende estreitamente da adesão do paciente $\mathrm{e}^{10,11}$. Segundo Fikri-Benbrahim et al. ${ }^{12}$, a não adesão é um fenômeno que constitui um grande problema para o tratamento, especialmente de doenças crônicas. Leite e Vasconcellos ${ }^{13}$ afirmam que o sucesso da terapia - a cura, controle ou prevenção de uma patologia - depende da adesão ao tratamento. Dessa forma, pode-se sugerir que um dos problemas encontrados no Brasil não seria a oferta de tratamento, mas a adequação das abordagens propostas, visando à maior adesão ao tratamento. Por conta disso, acreditamos na importância do estudo das características dessa população, a fim de possibilitar a melhor compreensão do fenômeno em questão.

Assim, este artigo tem o objetivo de comparar o perfil dos pacientes que aderiram e que não aderiram ao tratamento do CAPSad de Campo Grande (MS).

\section{MÉTODOS}

Realizou-se um estudo retrospectivo baseado no registro de prontuários, usando como material de coleta de dados os arquivos dos pacientes que deram entrada com APAC (Autorização de Procedimento de Alta Complexidade/Custo) por solicitação do CAPSad. A escolha desse material se deu por ser o melhor mecanismo à nossa disposição para a investigação proposta, uma vez que cada paciente que inicia o tratamento num CAPS gera uma APAC que é arquivada com todas as informações dele.

O arquivo de APACs do CAPSad de Campo Grande é organizado por ordem de entrada dos pedidos de tratamento. Cada registro é numerado para facilitar a localização. Dentro dessas pastas encontram-se, além das notas de APAC, a ficha de acolhimento, avaliação multiprofissional (psicólogo, psiquiatra, terapeuta ocupacional, professor de educação física, assistente social), dados de evolução do paciente e uma foIha que marca a frequência do paciente.

Desde o início das atividades do CAPSad de Campo Grande - acerca de três anos antes da pesquisa -, foram gerados pouco mais de 900 registros de APACs (o que representa o número de pacientes que efetivamente iniciaram o tratamento). Para a realização da pesquisa, foi sorteada de forma aleatória uma amostra de 330 registros, dos quais 316 foram utilizados e 14 foram excluídos.

Os critérios de inclusão para a pesquisa foram os seguintes: registros com ficha de acolhimento, avaliação psiquiátrica, notas de solicitação de APAC e ficha de frequência do paciente, pois os dados de interesse para a pesquisa encontram-se principalmente nesses documentos. Além disso, os registros deveriam ter numeração inferior a 851, uma vez que a maioria dos registros a partir da numeração supracitada era de pacientes que haviam iniciado o tratamento no CAPSad há menos de três meses, o que inviabilizava a nossa investigação.

Adotamos como critério de exclusão registros que, mesmo contendo os documentos exigidos para a inclusão, não continham a maior parte das informações de interesse da pesquisa.

Com base nos dados coletados, dividimos os pacientes em dois grupos: o grupo que aderiu ao tratamento 
$(G A ; N=176)$ e o grupo que não aderiu ao tratamento (GNA; $N=140$ ).

Usamos como critério para determinar a adesão a frequência por pelo menos três meses em uma das modalidades de tratamento oferecidas pelo CAPSad. O tempo de três meses foi escolhido como critério de adesão, pois cada APAC - material escolhido para a coleta dos dados - tem a duração de três meses, e para que uma APAC seja renovada é necessário que o paciente tenha permanecido em atendimento durante os três meses anteriores. Além disso, julgamos que três meses - dentro das possibilidades que tínhamos - era o tempo mínimo necessário para determinar se um paciente havia ou não aderido ao tratamento. Isso porque o CAPSad não adota um tempo de duração predeterminado para o tratamento, haja vista que, por a adicção ser uma doença crônica, seu alvo não é a "cura", mas a abstinência, o que requer um acompanhamento prolongado ${ }^{14}$.

Verificamos em ambos os grupos as seguintes características sociodemográficas: sexo, idade, registro de filiação (se foi registrado por pai e mãe ou apenas por mãe), se possui ocupação (trabalha ou estuda) e quantos anos de estudo tem.

As seguintes características clínicas também foram analisadas: droga de uso principal, número de tipos de drogas que já usou, idade em que iniciou o uso, tempo de uso, tratamentos anteriores para abandonar o uso de drogas, internações em decorrência do uso abusivo de álcool e outras drogas e tipo de tratamento que iniciou no CAPSad.

Esta pesquisa foi autorizada pela Instituição à qual estamos vinculados e pela Coordenação de Saúde Mental da Secretaria de Saúde de Campo Grande (MS). E, para a realização dela, baseamo-nos nos princípios éticos internacionais da declaração de Helsinki ${ }^{15}$.

\section{Análise dos dados}

As variáveis contínuas foram avaliadas quanto à sua média e desvio-padrão (DP), e as variáveis categóricas, quanto às frequências absolutas e relativas. Foi utilizado o teste $t$ de Student e ANOVA, para a comparação das variáveis contínuas entre os grupos, e o teste qui-quadrado, para a comparação das variáveis categóricas entre os grupos. Consideramos como associações estatisticamente significativas aquelas que apresentaram $p \leq 0,05$.

\section{RESULTADOS}

A amostra se constitui de 316 registros de pacientes do CAPSad de Campo Grande (MS), em sua maioria homens (86,4\%), com idade média de 35,2 anos ( $D P=13,7$ ), com registro de pai e mãe em seus documentos (88,0\%), sem ocupação $(51,5 \%)$ e com média de 7,2 anos de estudo (DP $=2,9)$. Em sua maioria, eram usuários de álcool $(48,7 \%)$ e faziam uso de uma média de 2,2 tipos de drogas ( $D P=1,2)$, tendo iniciado o uso de drogas em média aos 17,3 anos de idade ( $D P=6,9$ ), com média de 18,1 anos de uso (DP =13,3). Predominavam pacientes que nunca fizeram tratamentos anteriores para deixar o uso abusivo de álcool e outras drogas (62,0\%), nunca foram internados em consequência do uso abusivo de álcool e outras drogas $(63,9 \%)$ e realizaram tratamento não intensivo $(40,5 \%)$ no CAPSad.

Comparando o perfil sociodemográfico dos grupos, observou-se uma tendência de maior prevalência de pacientes do sexo masculino no $G A(89,8 \%$ versus $82,1 \% ; p=0,072)$. Os pacientes do GA eram mais velhos, já que tinham idade média de 37,0 anos ( $D P=14,0$ ) e os do GNA tinham idade média de 32,9 anos ( $D P=13,2)(p=0,008)$. Observamos, ainda, a tendência de maior prevalência de pacientes que foram registrados por pai e mãe no GA (91,5\% versus $84,2 \% ; p=0,068)$.

Já a comparação do perfil clínico dos grupos apresentou os seguintes dados: maior prevalência de usuários de álcool no $G A(p=0,005)$ (Figura 1) e maior tempo de uso de drogas no $G A, 20,0$ anos ( $D P=13,8$ ), contra 15,7 anos ( $D P=12,6$ ) do GNA $(p=0,005)$.

Fizemos, ainda, a verificação da idade em que os usuários de cada tipo de droga procuraram o atendimento no CAPSad e comparamos as diferenças etárias. Nessa parte da pesquisa, observamos que a idade média era de 41,3 anos $(\mathrm{DP}=11,9)$ para usuários de álcool, 32,7 anos (DP $=16,4)$ para usuários de tabaco, 28,0 anos ( $\mathrm{DP}=10,0)$ para usuários de cocaína e 24,0 anos ( $\mathrm{DP}=10,1)$ para usuários de maconha $(p=0,001)$. Na comparação da idade em que os usuários de cada tipo de droga procuraram tratamento, observamos diferença média de 17,3 anos $(p=0,000)$ entre usuários de álcool e maconha, 13,2 anos ( $p=0,000)$ entre usuários de álcool e cocaína, 8,6 anos ( $p=0,001)$ entre usuários de álcool e tabaco e 8,7 anos ( $p=0,003)$ entre usuários de tabaco e maconha.

Os dados estatisticamente significativos verificados em nossa pesquisa podem ser observados na tabela 1.

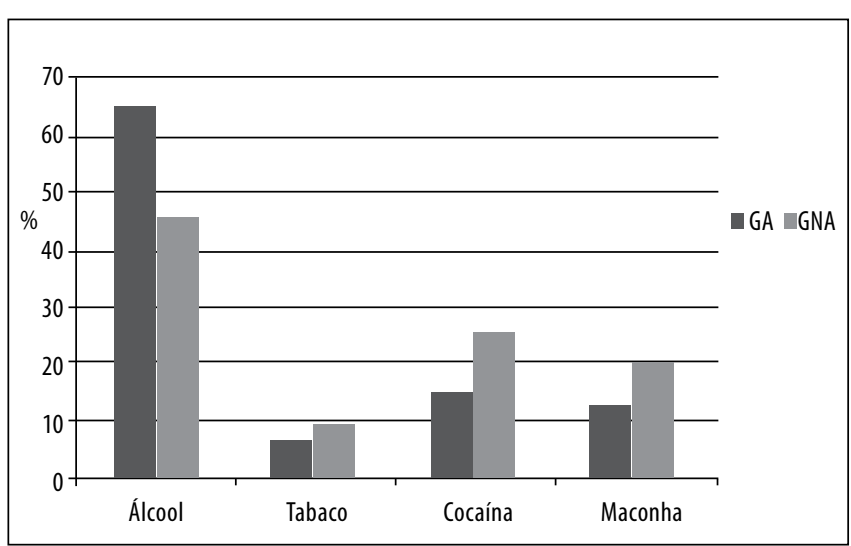

Figura 1. Indicação percentual da droga principal usada pelo grupo que aderiu (GA) e pelo grupo que não aderiu ao tratamento (GNA) - $\mathrm{p}=0,005$. 
Tabela 1. Comparação dos dados sociodemográficos e clínicos entre o grupo que aderiu (GA) ao tratamento e o grupo que não aderiu ao tratamento (GNA)

\begin{tabular}{|c|c|c|c|}
\hline & $G A(N=176)$ & GNA $(N=140)$ & Valor dep \\
\hline Sexo & & & 0,072 \\
\hline Masculino & $89,8 \%$ (158) & $82,1 \%(115)$ & \\
\hline Feminino & $10,2 \%(18)$ & $17,8 \%(25)$ & \\
\hline Idade & $37,0(D P=14,0)$ & $32,9(\mathrm{DP}=13,2)$ & 0,008 \\
\hline Registro de filiação* & & & 0,068 \\
\hline Pai e mãe & $91,5 \%(161)$ & $84,2 \%(117)$ & \\
\hline Somente mãe & $8,5 \%(15)$ & $15,8 \%(22)$ & \\
\hline Droga principal & & & 0,005 \\
\hline Álcool & $65,3 \%(115)$ & $45,3 \%(63)$ & \\
\hline Tabaco & $6,8 \%(12)$ & $9,4 \%(13)$ & \\
\hline Cocaína & $15,3 \%(27)$ & $25,9 \%(36)$ & \\
\hline Maconha & $12,5 \%(22)$ & $19,4 \%(27)$ & \\
\hline Tempo de uso** & $20,0(D P=13,8)$ & $15,7(D P=12,6)$ & 0,005 \\
\hline
\end{tabular}

\section{DISCUSSÃO}

Observamos em nossa amostra a prevalência de pacientes do sexo masculino em ambos os grupos, com tendência de maior prevalência de pacientes do sexo masculino no GA (89,8\% versus 82,1\%; $p=0,072)$. A prevalência geral de homens encontrada em nossa amostra está de acordo com Siqueira et al. ${ }^{16}$, que encontraram, em sua amostra de 216 pacientes do Programa de Atendimento ao Alcoolista do Hospital Universitário Cassiano Antônio Moraes da Universidade Federal do Espírito Santo, 88,5\% de pacientes do sexo masculino.

A tendência de maior prevalência de pacientes do sexo feminino no GNA (17,8\% versus 10,2\%; $p=0,072)$ pode ser explicada pelo exposto por Siqueira et al. ${ }^{16}$ e Fotin et al. ${ }^{17}$, que afirmam em seus estudos que mulheres procuram menos os serviços de atendimento a usuários de álcool e drogas do que os homens, por razões como o estigma social em relação ao papel da mulher e por conta de o próprio perfil de usuário da mulher ser diferente do homem. Cesarr ${ }^{18}$, em estudo qualitativo realizado com mulheres da Unidade de Tratamento de Alcoolismo do Instituto Philippe Pinel no Rio de Janeiro, observou que 90\% das mulheres declararam beber em âmbito privado e que se comportam de forma diferente quando bebem no âmbito social. Dado que pode indicar que mulheres conseguem esconder a dependência por mais tempo e que, por conta da forma como a sociedade vê a mulher alcoolista, há maior preocupação em relação à autoimagem ao expor-se a um tratamento em que terá que se deparar com outras pessoas. Fato esse que ganha mais consistência quando se leva em consideração que até bem pouco tempo antes de nossa pesquisa o CAPSad de Campo Grande não contava com um programa diferenciado para atendimento de grupos de mulheres.

Em nosso estudo verificamos que os pacientes do GA tinham idade média de 37,0 anos contra a idade média de $32,9$ anos do GNA ( $p=0,008)$, dado também observado por Burns et al. ${ }^{19}$, porém em uma amostra de pacientes em tratamento de substituição de opiáceos. Mesmo avaliando pacientes com um maior perfil de gravidade, os autores identificaram que indivíduos mais jovens foram significativamente mais propensos a abandonar o tratamento.

Outro dado encontrado em nosso estudo indica a tendência de maior prevalência de pacientes que foram registrados por pai e mãe no GA $(91,5 \%$ versus $84,2 \% ; p=0,068)$. Em relação a essa informação, Goeb et al. ${ }^{20}$ observaram, em estudo com 257 pacientes do Centro de Cassini em Paris, que os pacientes que possuem familiares envolvidos no programa de tratamento apresentam resultados mais favoráveis que os demais. O que encontra apoio em Schenker e Minayo ${ }^{21}$, que descrevem diversos métodos que se caracterizam essencialmente por intervenções que envolvem a família do adicto, destacando-se, assim, a importância da família no auxílio à adesão ao tratamento da adicção. Isso nos leva a especular sobre uma possível relação entre o registro por pai e mãe e uma melhor dinâmica familiar, capaz de apoiar o tratamento e, consequentemente, possibilitar maior tendência à adesão. Contudo, a correlação entre registro de nascimento e convívio familiar precisa de mais dados que a sustentem, não só do ponto de vista estatístico, como também teórico e empírico.

Nosso estudo também apontou para maior prevalência de usuários de álcool no $G A(p=0,005)$, diferente do que ocorreu com os usuários das demais drogas, que se encontravam em sua maioria no GNA. Esse dado pode ser compreendido quando se verifica que a idade média desses usuários era de 41,3 anos $(p=0,001)$ quando procuraram tratamento, e, por serem mais velhos do que os usuários de outros tipos de drogas, têm mais probabilidade de adesão ${ }^{19}$. Além disso, não se pode perder de vista que o uso de drogas lícitas está menos ligado ao comportamento antissocial e criminoso do que o uso de drogas ilícitas, o que pode ser um fator positivo para a adesão ao tratamento ${ }^{14,22}$.

Outro dado clínico encontrado em nossa pesquisa indica que os pacientes do GA têm mais tempo de uso do que os do GNA, sendo a idade média do primeiro 20,0 anos e a do segundo, $15,7(p=0,005)$. Segundo Souza e Siqueira ${ }^{23}$, $82,3 \%$ dos pacientes iniciam o uso de drogas na adolescência, com a idade média de 16,8 anos. Em outro estudo, Ferreira Filho et al. ${ }^{24}$ observaram entre os dependentes químicos residentes na Grande São Paulo que a idade média de início do uso de drogas era de 14,6 (DP = 3,3). Em nossa pesquisa, a idade média de início de uso dos pacientes foi de $17,3(\mathrm{DP}=6,9)$. Isso nos leva a concluir que, se no $\mathrm{GA}$ os 
pacientes são mais velhos do que no GNA e se a maioria dos usuários de drogas inicia o uso na adolescência, é natural que os pacientes do GA tenham mais tempo de uso do que os do GNA.

\section{CONCLUSÃO}

Findamos ressaltando que, apesar das limitações deste estudo feito por meio de análise de prontuários, pudemos observar que o paciente com maior dificuldade em aderir ao tratamento é mais jovem, com idade média 32,9 anos (DP = $13,2)(p=0,008)$, faz uso de drogas ilícitas, tem menos tempo de uso, em média 15,7 anos ( $D P=12,6)$, e, aparentemente, menor estruturação familiar. Assim, constatamos que as abordagens utilizadas atualmente pelo CAPSad têm contemplado quase exclusivamente homens, mais velhos, etilistas, com longo histórico de uso, oriundos de famílias com possível melhor dinâmica familiar. Uma vez que as práticas vigentes têm se mostrado pouco eficazes para os usuários com o perfil de não adesão, salientamos a importância de implantar abordagens diferenciadas e mais precoces, que ajudem o usuário a ser tratado antes de ter a vida produtiva prejudicada pela dependência. Para isso, talvez seja interessante uma busca mais ativa por parte de governos e instituições de saúde mental como o CAPSad, um estreitamento em suas relações com outros programas de saúde como o Programa de Saúde da Família (PSF) e, por que não, também o estreitamento de relações com outras instituições ligadas à comunidade como escola, centros comunitários e igrejas', a fim de criar uma rede de apoio para os usuários em todos os lugares em que ele esteja interagindo e, principalmente, auxiliar a família e a comunidade a perceberem ainda cedo a necessidade de tratamento. É preciso, ainda, maior investimento por parte do governo para que o CAPSad esteja inserido nas comunidades como preconiza sua estratégia ${ }^{7}$, pois ainda hoje o CAPSad atua muito mais como um instrumento ambulatorial do que propriamente comunitário.

\section{REFERÊNCIAS}

1. Pinho PH, Oliveira MA, Almeida MM. A reabilitação psicossocial na atenção aos transtornos associados ao consumo de álcool e outras drogas: uma estratégia possível? Rev Psiq Clín. 2008:35:82-8

2. Meloni JN, Laranjeira R. Custo social e de saúde do consumo do álcool. Rev Bras Psiq. 2004;26:7-10.
3. Galduróz JCF, Caetano R. Epidemiologia do uso de álcool no Brasil. Rev Bras Psiq. 2004;26:3-6.

4. Brasil. Decreto no 6.117, de 22 de maio de 2007. Aprova a Política Nacional sobre o Álcool, dispõe sobre as medidas para redução do uso indevido de álcool e sua associação com a violência e criminalidade, e dá outras providências. Diário Oficial da União 23 maio 2007.

5. Brasil. Ministério da Saúde. Secretaria de Atenção à Saúde. SVS/CN-DST/AIDS. A Política do Ministério da Saúde para Atenção Integral a Usuários de Álcool e outras Drogas/Ministério da Saúde. 2. ed. rev. ampl. Brasília: Ministério da Saúde; 2004.

6. Braślia. Primeira Conferência Pan-Americana de Políticas Públicas Sobre Álcool; 2005

7. Brasil. Ministério da Saúde. Secretaria de Atenção à Saúde. Departamento de Ações Programáticas Estratégicas. Saúde mental no SUS: os centros de atenção psicossocial. Brasília; 2004.

8. Moraes E, Campos GM, Silva SP, Figlie NB, Laranjeira R. Visita domiciliar no tratamento de pacientes dependentes de álcool: dados preliminares. Rev Bras Psiq. 2005;27:347-8.

9. Scaduto AA, Barbieri V. 0 discurso sobre a adesão de adolescentes ao tratamento da dependência química em uma instituição de saúde pública. Ciênc Saúde Coletiva. 2009:14:605-14

10. Llorca PM, Miadi Fargier-H, Laçon C, Mosqueda JG, Casadebaig F, Philippe A, et al. Cost-effectiveness analysis of schizophrenic patient care settings: impact of an atypical antipsychotic under long-acting injection formulation. Encephale. 2005;31(2):235-46.

11. Rabenda V, Reginster JY. Positive impact of compliance to strontium ranelate on the risk of nonvertebral osteoporotic fractures. Osteoporos Int. 2010;16.

12. Fikri-Benbrahim N, García-Cárdenas V, Sáez-Benito L, Gastelurrutia MA, Faus MJ. Adherence: a review of education, research, practice and policy in Spain. Pharmacy Practice. 2009:7(3):125-38.

13. Leite SN, Vasconcellos MPC. Adesão à terapêutica medicamentosa: elementos para a discussão de conceitos e pressupostos adotados na literatura. Ciênc Saúde Coletiva. 2003;8(3):775-82.

14. Sadock BJ, Sadock VA. Compêndio de psiquiatria: ciência do comportamento e psiquiatria clínica. 9. ed. Porto Alegre: Artmed; 2007, p 419.

15. Diniz D, Corrêa M. Declaração de Helsinki: relativismo e vulnerabilidade. Cad Saude Publica. 2001;17(3):679-88

16. Siqueira MM, Garcia MLT, Souza RS. 0 impacto das faltas às consultas em um programa de dependentes de álcool. J Bras Psiquiatr. 2005;54(2):114-9.

17. Fotin RMP, Libre AT, Mello AF. Estudo da morbidade do alcoolismo na Santa Casa de Sao Paulo. Rev ABP-APAL. 1995;17(2):67-71.

18. Cesar BAL. Alcoolismo feminino: um estudo de suas peculiaridades. Resultados preliminares. J Bras Psiquiatr. 2006;55:208-11.

19. Burns L, Randall D, Hall WD, Law M, Butler T, Bell J, et al. Opioid agonist pharmacotherapy in New South Wales from 1985 to 2006: patient characteristics and patterns and predictors of treatment retention. Addiction. 2009;104(8):1363-72.

20. Goeb JL, Coste J, Bigot T, Ferrand I. Prospective study of favorable factors in follow-up of drug addicted patients - apropos of 257 patients of the Cassini Center in Paris. Encephale. 2000;26(6):11-20.

21. Schenker M, Minayo MCS. A importância da família no tratamento do uso abusivo de drogas: uma revisão da literatura. Cad Saude Publica 2004;20(3):649-59.

22. Heim J, Andrade AG. Efeitos do uso do álcool e das drogas ilícitas no comportamento de adolescente de risco: uma revisão das publicações científicas entre 1997 e 2007. Rev Psiq Clín. 2008;35(1):61-4.

23. Souza RS, Siqueira MM. 0 processo de enfermagem na assistência a pacientes com dependência de álcool. J Bras Psiquiatr. 2005;54:228-33.

24. Ferreira Filho OF, Turchi MD, Laranjeira R, Castelo A. Perfil sociodemográfico e de padrões de uso entre dependentes de cocaína hospitalizados. Rev Saude Publica. 2003;37:751-9. 